\title{
Wide spectrum modulation by KP-544 in models relevant for neuronal survival
}

\author{
Marie A. Geist, Christiane Volbracht, Jana Podhorna, Jeppe Falsig and Marcel Leist \\ H. Lundbeck A/S, Department of Disease Biology, Valby, Denmark \\ Correspondence and requests for reprints to Marie A. Geist, PhD, H. Lundbeck A/S, Ottiliavej 9, 2500 Valby, Denmark \\ Tel: + 453643 2380; fax: + 453643 8903; e-mail: maag@lundbeck.com
}

Current address: Marcel Leist, Department of Biology, University of Konstanz, Konstanz, Germany; Jeppe Falsig, Institute of Neuropathology, University Hospital Zurich, Switzerland.

\begin{abstract}
Reduced neurotrophic signalling has been proposed as a part of the pathophysiology behind neuronal death and dysfunction. The small molecule KP 544 was developed with the intention to enhance nerve growth factor signalling. To characterize the actions of KP 544 pharmacologically, we used four diverse models with relevance for neuronal function and survival. We found that $3001000 \mathrm{nM}$ KP 544 enhanced the neurite outgrowth in $\mathrm{PCl} 2$ cells in response to a suboptimal concentration of nerve growth factor. KP 544
\end{abstract}

also protected the cerebellar granule cells from excitotoxicity apoptosis induced by the mitochondrial toxin methyl phenyl pyri dinium, and modulated inflammation by inhibiting interleukin 6 production in primary astrocytes. Chronic treatment of rats with KP 544 prevented the hyper responsiveness to amphetamine of animals treated with methylazoxymethanol acetate, a recently described neurodevelopmental model of schizophrenia. NeuroReport 18:57| 575 (c) 2007 Lippincott Williams \& Wilkins.

Keywords: excitotoxicity, inflammation, methylazoxymethanol acetate, neurite outgrowth, neuronal cell death, neurotrophin enhancement

\section{Introduction}

Functional impairment and degeneration of neurites, inflammation, and cell death are common features of many neurodegenerative disorders [1], and lack of neurotrophic signalling has been proposed as a part of the underlying pathophysiology [2]. Recently, psychiatric disorders have also been associated with neuronal atrophy. Such disturbances have been associated with perturbations during development, changed neurotrophin levels in serum, and altered receptor levels in patients with psychiatric disorders [3]. Neurotrophins regulate the plasticity by promoting neurite outgrowth [4], and promote functional restoration [5]. In models of neurodegeneration, neurotrophins are protective [4], suggesting that activation of neurotrophic signalling pathways may be a beneficial treatment for neurodegenerative diseases and psychiatric disorders. Exogenous application of neurotrophic factors, however, has been unsuccessful as yet, because of difficulties in delivering the relatively large proteins to the target cells and because of severe side effects [6]. KP-544 is a novel small molecule, reported to affect nerve growth factor (NGF) signalling, possibly at a level downstream of mitogen-activated protein kinases [7]. Here, we investigated the possible beneficial effects of KP-544 in in vitro models, representing different aspects of dysfunction in the nervous system, namely neurite outgrowth, neuronal death, and inflammation. Furthermore, we studied the possible beneficial effects of KP-544 in the neurodevelopmental methylazoxymethanol acetate (MAM) model of schizophrenia [8].

\section{Materials and methods}

KP-544 (trans-4-[[2-amino-5-[(4-chlorophenyl)ethynyl]-4pyrimidinyllamino]cyclohexanol) was kindly provided by Krenitsky Pharmaceuticals Inc. (Durham, North Carolina, USA). All other reagents and antibodies were purchased from Sigma-Aldrich (St Louis, Missouri, USA), unless otherwise stated.

PC12 cells were maintained in Dulbecco's modified Eagle's medium supplemented with $10 \%$ heat-inactivated horse serum, 5\% heat-inactivated fetal calf serum, $1 \%$ sodium pyruvate, and $1 \%$ penicillin streptomycin (all purchased from Gibco-BRL Invitrogen, San Diego, California, USA). Cells were plated in 96-well plates at 1500 cells / well in growth medium and treated with NGF (2.5S) or $342 \mathrm{nM}$ KP-544 either alone or together with $10 \mathrm{ng} / \mathrm{ml} \mathrm{NGF}$, with medium change and new addition of compounds 3 times/week. $342 \mathrm{nM}$ was chosen as it corresponds to $1 \mathrm{mg} /$ $\mathrm{ml}$ found in earlier studies to enhance NGF signalling [7]. After 7 days, the cells were fixed in 3.7\% formaldehyde and immunostained for tubulin using the antibody provided in the 'neurite outgrowth' kit (Cellomics, Pittsburgh, Pennsylvania, USA). The nuclei were visualized with H-33342 also provided in the kit. The neurite outgrowth index (the percentage of neurons with a summed neurite length longer than $20 \mu \mathrm{m}$ ) was determined by image processing software using the 'neurite outgrowth' algorithm on the chargecoupled device camera-based ArrayScan HCS system (Cellomics). Forty microscopic fields were analysed for one data point. 
Murine cerebellar granule cells (CGCs) were isolated and cultured as described previously [9]. The neurons were plated in poly-L-lysine-coated 24-well plates at 1800000 cells $/ \mathrm{ml}$. After $48 \mathrm{~h}, 10 \mu \mathrm{M}$ cytosine arabinoside was added. At 7 days in vitro, cells were preincubated for 30 min with different concentrations of KP-544, before $25 \mu \mathrm{M}$ of 1-methyl-4-phenylpyridinium $\left(\mathrm{MPP}^{+}\right)$was added to the culture. After $6 \mathrm{~h}$, cell death was quantified on the population level by using 3-(4,5-demethylthiazole-2-yl)-2,5diphenyltetrasodium bromide-reduction assay and at $6+24 \mathrm{~h}$ by evaluation of neuronal morphology in phasecontrast and after H-33342 staining. Alternatively, to assess the plasma membrane integrity and nuclear morphology, CGC were double stained with the chromatin dyes SYTOX $(0.5 \mu \mathrm{M}$, noncell permeable, green fluorescent) and $\mathrm{H}-33342$ $(1 \mu \mathrm{g} / \mathrm{ml}$, cell permeable, blue fluorescent). All dyes were obtained from Molecular Probes (Eugene, Oregon, USA). Apoptosis was characterized by scoring condensed and highly fluorescent nuclei. About 300 cells were counted in three different fields in three different culture wells, and experiments were repeated in at least three different preparations.

Primary cortical astrocytes were isolated and prepared as described previously [10]. Cells were trypsinized and reseeded (at 10000 cells/well in a 96-well plate) after 14 days in culture and were always used for experiments 68 days after replating. The cells were preincubated with KP$54430 \mathrm{~min}$ before the addition of a cytokine mix (interferon $\gamma 5 \mathrm{U} / \mathrm{ml}$, tumour necrosis factor- $\alpha 10 \mathrm{ng} / \mathrm{ml}$, interleukin- $1 \beta$ (IL- $\beta) 10 \mathrm{ng} / \mathrm{ml}$ ) and after $24 \mathrm{~h}$ the release of IL-6 was measured using a murine-specific OptEIA ELISA kit (BD Pharmingen, Brøndby, Denmark). Cell death was measured using lactate dehydrogenase release assay in parallel wells.

MAM was supplied by the National Cancer Institute and was dissolved in $0.9 \% \mathrm{NaCl}$. KP-544 was dissolved in $10 \%$ cyclodextrine. Dexamphetamine sulphate was supplied by Nomeco (Copenhagen, Denmark) and was dissolved in $0.9 \% \mathrm{NaCl}$. Control groups received the appropriate vehicle. All animal experiments were performed in accordance with the guidelines of the Danish National Committee on Animal Research Ethics and the European Communities Council Directive \#86/609 for the Care of Laboratory Animals.

The animals were treated with MAM on gestation day 17, exactly as described previously in [8]. Male offspring of vehicle-treated or MAM-treated dams were assigned to chronic treatment with KP-544 $(1 \mathrm{mg} / \mathrm{kg}$ daily per orally, $10 \mathrm{ml} / \mathrm{kg}$ ) or vehicle ( $10 \%$ cyclodextrine). Treatment started on postnatal day 40 and continued for 5 weeks.

Twelve hours after the last injection of KP-544 (postnatal day 74 75), locomotor activity of rats was assessed in large open field arenas $\left(150 \times 100 \times 40 \mathrm{~cm}^{3}\right)$. The behaviour of rats was recorded by a video camera that was placed above the arena. Testing was performed during dark phase and the room was illuminated with bright light (stress condition). Rats were injected with saline, placed in the centre of the arena, and their activity was measured for $30 \mathrm{~min}$ (habituation + basal activity). The rats were then removed, given an injection of amphetamine ( $2 \mathrm{mg} / \mathrm{kg}$ subcutaneous, $5 \mathrm{ml} / \mathrm{kg}$ ), and placed back in the arena for $120 \mathrm{~min}$. A blinded observer scored the videotapes. The open field arena was divided into 12 identical squares and the number of line crossing was recorded for each rat. Data were analyzed using repeated two-way analysis of variance with factors group and challenge (saline or amphetamine). As locomotor activity after saline was measured for $30 \mathrm{~min}$ whereas after amphetamine for $120 \mathrm{~min}$, the number of crossings after amphetamine was divided by 4 . Student Newman Keuls pairwise multiple post-hoc comparison method was used to compare groups within each challenge.

\section{Results}

Maintenance of the axodendritic network is an approach to treat the neurodegenerative diseases. We initially tested whether KP-544 was able to affect the NGF-driven neurite outgrowth in PC12 cells. Cells were cultured in the presence of KP-544 (342 nM), either alone or together with NGF, and the neurite outgrowth index was determined. NGF $(10 \mathrm{ng} / \mathrm{ml})$ treatment alone led to a slight increase in neurite outgrowth compared with untreated controls (Fig. 1a). KP544 treatment alone had a minimal effect on neurite outgrowth (Fig. 1a). KP-544 together with a sub-optimal concentration of NGF $(10 \mathrm{ng} / \mathrm{ml})$ enhanced neurite outgrowth compared with either treatment alone (Fig. 1a) and significantly increased neurite outgrowth index (Fig. 1b). Then we examined whether KP-544 increased the number of outgrowing neurites or the neurite length. The number of neurites was similar in cells stimulated with NGF alone or NGF and KP-544 (Fig. 1c), suggesting that the neurite length was increased in cells stimulated with KP-544. This was confirmed by a quantitative manual counting and morphometry study. We conclude from these results that KP-544 has NGF-dependant neurotrophic effects in the higher nanomolar range in vitro.

Next, we investigated whether KP-544 had direct neuroprotective ability. We used the well established model system of CGCs challenged with the neurotoxin $\mathrm{MPP}^{+}$. $\mathrm{MPP}^{+}$, an inhibitor of the mitochondrial respiratory chain complex I [11], has been shown to impair the mitochondrial function and to lead to energy failure, triggering endogenous glutamate release followed by the loss of intracellular $\mathrm{Ca}^{2+}$ homeostasis and finally excitotoxicity in CGCs [9]. CGCs were treated with $\mathrm{MPP}^{+}(25 \mu \mathrm{M})$ in the presence or absence of $\mathrm{KP}-544(0.1,1,10 \mu \mathrm{M})$ and viability was quantified on the population level by 3-(4,5-demethylthiazole-2-yl)-2,5-diphenyltetrasodium bromide-reduction (Fig. 2). In addition, apoptotic cells characterized by nuclear morphology and integrity were counted individually and the results of both assays were in good agreement (data not shown). KP-544 (1 and $10 \mu \mathrm{M})$ completely protected from $\mathrm{MPP}^{+}$-induced neuronal death, suggesting that KP-544 is neuroprotective against excitotoxicity in the $1 \mu \mathrm{M}$ range.

Inflammation and reactive astrocytes are associated with neurodegenerative diseases [12] as evidenced by increased levels of cytokines [13]. We investigated whether KP-544 was able to modulate an inflammatory response in vitro. Primary astrocytes were treated with a cytokine mix to induce IL-6 release, which was measured as a marker for inflammation in the absence or presence of KP-544 (1, 3, and $5 \mu \mathrm{M})$. KP-544 significantly decreased the cytokine mixinduced IL- 6 release from the astrocytes at 3 and $5 \mu \mathrm{M}$ (data not shown; $P<0.05$, one-way analysis of variance followed by the Tukey test), suggesting that KP-544 has an antiinflammatory capacity on astrocytes in vitro in the low micromolar range. To confirm that the decreased IL-6 release was not due to increased cell death, the lactate dehydrogenase released was measured. KP-544 did not induce cell death in these cultures (data not shown). 
(a)
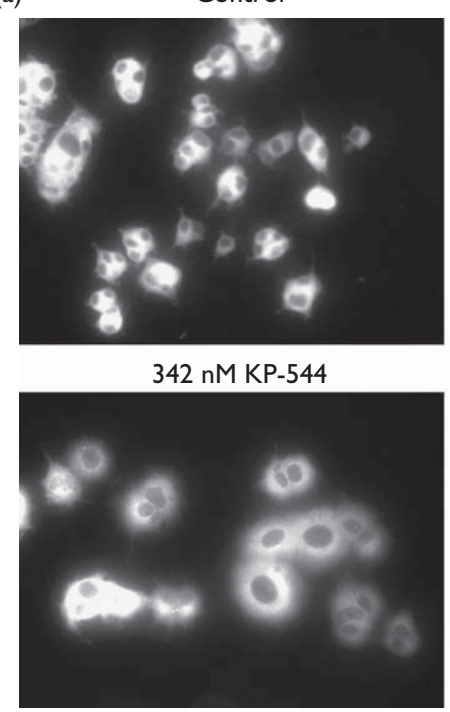

$10 \mathrm{ng} / \mathrm{ml} \mathrm{NGF}$

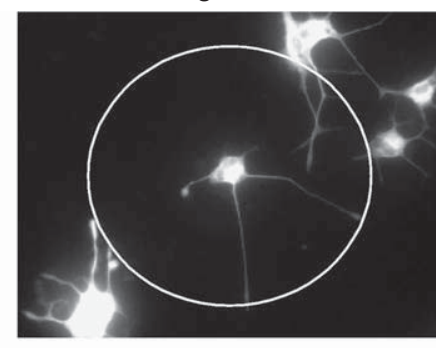

KP-544 + NGF

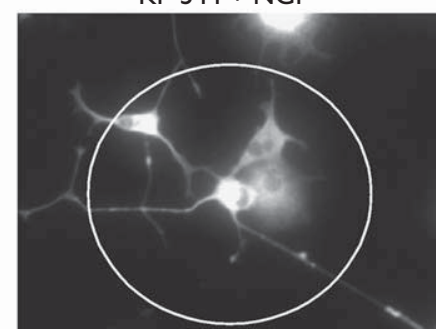

(b)

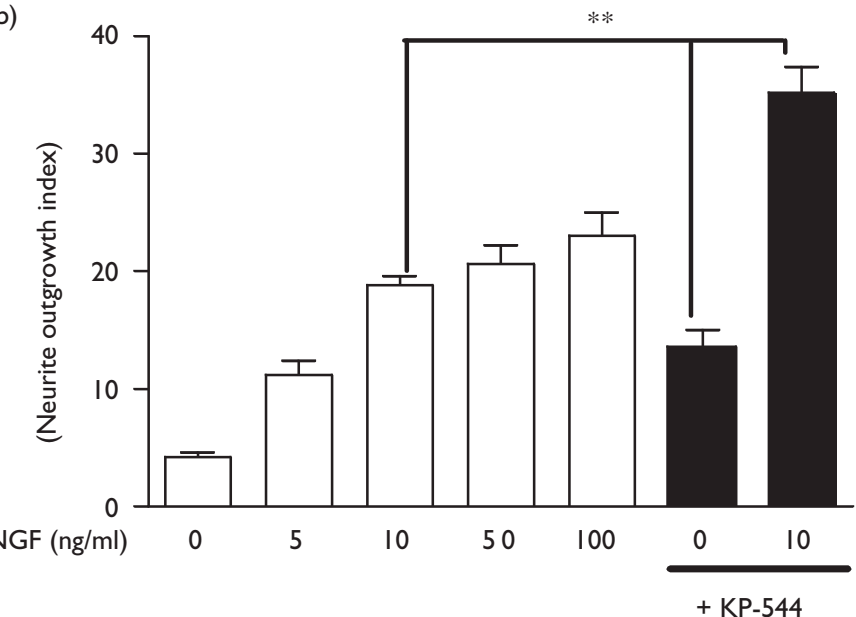

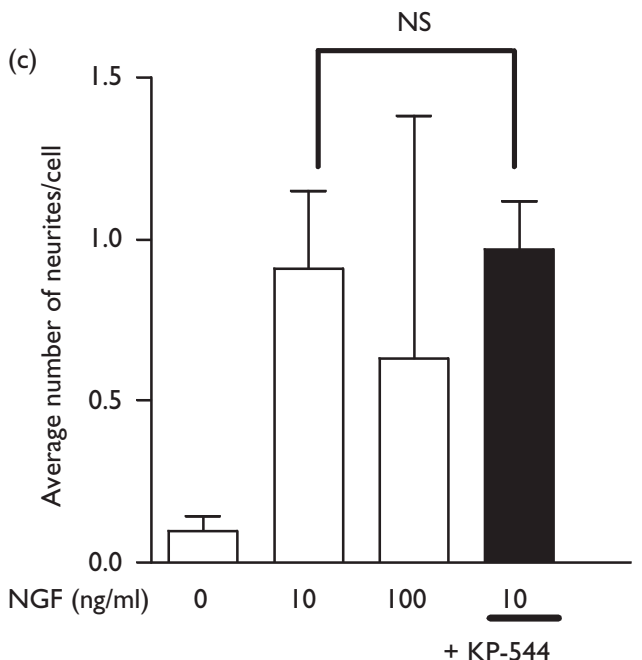

Fig. I KP-544 promotes neurite outgrowth. PCI2 cells treated with nerve growth factor (NGF) only, or with KP-544 (342 nM) in the presence or absence of $10 \mathrm{ng} / \mathrm{ml}$ NGF. After 7 days, the cells were fixed and immunostained for tubulin. Nuclei were visualized with Hoechst-33342. (a) Pictures are representative of three experiments. The white rings mark the size of an average cell, including neurites, treated with $10 \mathrm{ng} / \mathrm{ml} \mathrm{NGF}$. Image width corresponds to $220 \mu \mathrm{m}$. (b) Neurite length was determined by automatic digital image analysis of the staining outside the cell bodies. Data are presented as neurite outgrowth index (percentage of neurons whose summed neurite lengths are greater than $20 \mu \mathrm{m}$ ). Data represent quantifications from $n \quad 4$ wells with minimum 10 fields/well (mean $\pm S E M)(* * P<0.0$ l, one-way analysis of variance followed by theTukey test). (c) The number of neurites was determined by counting the neurites with a length greater than the diameter of the cell body in the same cultures.

Finally, we investigated the effects of KP-544 in a recently described neurodevelopmental model of schizophrenia, in which the mitosis inhibitor MAM, given to pregnant rats on gestation day 17, disrupts the neurogenesis of embryos transiently. Offspring from MAM-treated mothers displays several characteristics of the disease such as changed neurotrophin levels [14] and increased sensitivity to amphetamine [7]. To test the possible restorative effect of $\mathrm{KP}-554$ in vivo, we treated animals with $1 \mathrm{mg} / \mathrm{kg} \mathrm{KP}-544$ for 5 weeks, starting at postnatal day 40 , before subjecting the animals to amphetamine-induced hyperactivity at postnatal day $74 / 75$. Activity, measured as locomotion, was similar in all four groups after saline challenge (data not shown). As expected, amphetamine treatment significantly increased locomotion in all the groups of rats $[F(1,57)=138.5$, $P<0.001$ ] (Fig. 3). Importantly, amphetamine-induced hyper- activity was significantly increased in MAM animals compared with control animals (Fig. 3) in the non-KP544 groups. Treatment with KP-544 lowered this increased hyperactivity in MAM animals by around 50\% (Fig. 3), although the overall statistical analysis did not reveal significant group difference $[\mathrm{F}(3,57)=2.02$, NS]. KP-544-treated MAM animals, however, were also not significantly different from vehicle-treated control or MAM alone animals. Thus, the observed effect of KP-544 may indicate a reversal of the increased amphetamine hypersensitivity seen in the MAM-treated rats, but larger experiments are required to confirm these data statistically.

\section{Discussion}

We have shown that the novel small molecule KP-544 has positive effects on neuronal function and survival in vitro. 


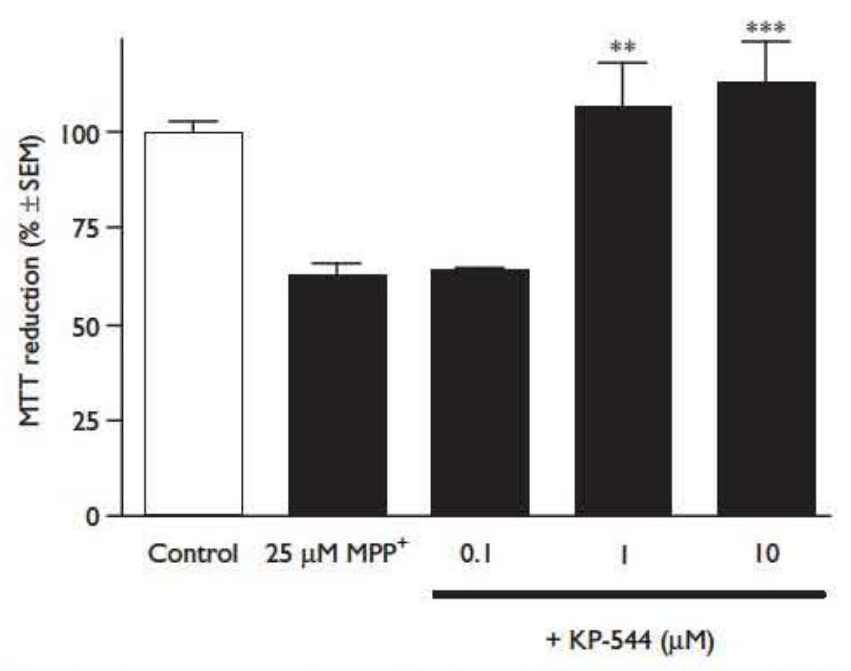

Fig. 2 Protection from cytotoxicity in cerebellar granule cells. CGCs were stimulated with $25 \mu \mathrm{M}$ methyl-phenyl-pyridinium $\left(\mathrm{MPP}^{+}\right)$in the presence or absence of KP-544. Viability was measured after $6 \mathrm{~h}$ by 3-(4,5-demethylthiazole-2-yl)-2,5-diphenyltetrasodium bromide (MTT)reduction assay. Data are presented as percentage of control cells (mean \pm SEM), $n \quad 3 .{ }^{*} * P<0.01$; ${ }^{* * *} P<0.001$, one-way analysis of variance followed by the Tukey test.

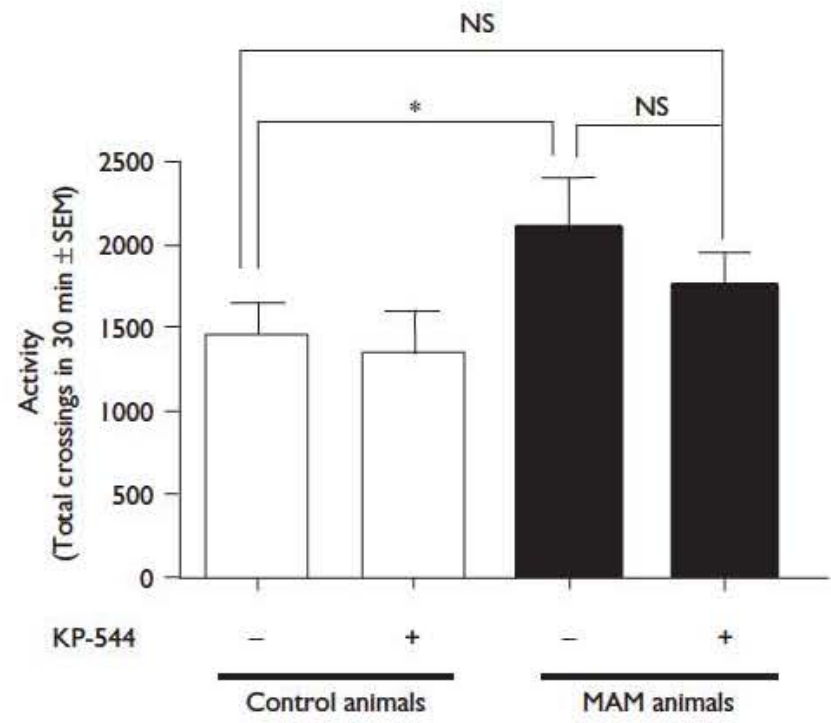

Fig. 3 Effects of KP-544 on amphetamine-hyperactivity in vivo. Male Wistar rats originating either from vehicle (control animals) or methylazomethanol acetate (MAM animals)-treated dams were treated with $\mathrm{KP}-544(1 \mathrm{mg} / \mathrm{kg})$ or vehicle for 5 weeks starting at postnatal day 40 . Amphetamine hyperactivity was assessed on postnatal day $74 / 75$. Animals were treated with saline and consecutively with $2 \mathrm{mg} / \mathrm{kg}$ of a mphetamine and the number of line crossings was measured and scored as activity ( \pm SEM). ${ }^{*} P<0.05$; repeated two-way analysis of variance followed by the Student Newman Keuls test; NS, not significant.

KP-544 increased neurite outgrowth in response to a suboptimal concentration of NGF. These results support a previous report from Fyfe et al. [7] showing that KP-544 enhanced NGF signalling. We show here that KP-544 protected concentration-dependently from $\mathrm{MPP}^{+}$-induced indirect excitotoxicity, which is independent of NGF signalling [9]. Excitotoxicity is thought to contribute to a broad variety of neurological diseases [15] with energy depletion among the frequent initiating conditions leading to excitotoxicity. Mitochondrial dysfunction is believed to be one of the most generalized conditions favoring the development of neurodegenerative diseases [16]. Our results suggest that KP-544's neuroprotective property is different from the NGF-enhancing effect, and might be relevant to excitotoxicity in neurodegenerative diseases. Further, we showed that KP-544 affected astrocytes by attenuating the release of IL-6, suggesting that KP-544 has anti-inflammatory abilities in vitro as a third biological activity. Astrocytes are important for maintaining homoeostasis in the brain by taking up excess neurotransmitters and secreting different survival-promoting factors. They are, however, implicated in neurodegenerative processes by their ability to release proinflammatory cytokines [10]. Increased levels of the proinflammatory IL- 6 are implicated in several neurodegenerative diseases [17], and in experimental models of neurodegeneration [18]. Our results show that KP-544 was able to inhibit the release of the proinflammatory cytokine IL-6 in vitro, indicating that KP544 might be neuroprotective, indirectly by affecting astrocytes in addition to its direct effect on neuronal survival. Whether KP-544 has a more general dampening effect on inflammation remains to be investigated. Altogether, we show here that in vitro KP-544 had enhancing effects on neurite outgrowth dependent on NGF, and NGFindependent neuroprotective and anti-inflammatory abilities in the low micromolar range. This concentration, resulting in significant protection is, in fact, in the concentration range that can be achieved in the brain. Previous pharmacokinetic data have revealed that a single oral dose of $10 \mathrm{mg} / \mathrm{kg} \mathrm{KP-544}$ results in plasma levels of $1 \mu \mathrm{M}$ and six-fold higher levels in the brain [19]. KP-544 has been shown previously to be beneficial in acute injury models in vivo, which were dependent on NGF deficiency. KP-544 completely reversed the learning deficits induced by intrastriatial quiolinic acid lesions in rats and decreased taxol-induced peripheral neuropathy in mice [19]. Here, we used KP-544 in a chronic disease model, the neurodevelopmental MAM model of schizophrenia, in which rats were treated with MAM on gestation day 17. Offspring from MAM-treated mothers shows several aspects of the human disease, such as atrophy of the hippocampus, thalamus, and cortical regions, cognitive impairment such as in reversal learning, and increased sensitivity to psychostimulants $\left[\begin{array}{ll}7,20 & 23\end{array}\right]$. In addition, NGF levels are decreased in several of the relevant brain regions such as the entorhinal cortex and hippocampus [14,24]. Interestingly, chronic treatment with KP-544 was able to partly improve the hypersensitivity to amphetamine seen in MAM-treated animals. The observed effect, however, can only be described as a trend as it was not significant on stringent statistical analysis. Our data indicate that KP-544 might have beneficial effects in a real disease setting, where slowly progressive neurodegeneration requires chronic treatment.

\section{Conclusion}

We show in this study for the first time that the small molecule KP-544 is able to enhance NGF-induced neurite growth and has neuroprotective and anti-inflammatory abilities in vitro. KP-544 may provide an effective strategy to reduce neuronal death and inflammation, and to promote 
neuronal survival in pathological conditions linked to impaired neurotrophin signalling in neurodegenerative and psychiatric disorders.

\section{Acknowledgements}

We would like to thank Krenitsky Pharmaceuticals Inc. (Durham, North Carolina, USA) for generously providing KP-544.

\section{References}

1. Pereira C, Agostinho P, Moreira PI, Cardoso SM, Oliveira CR. Alzheimer's disease-associated neurotoxic mechanisms and neuroprotective strategies. Curr Drug Targets CNS Neurol Disord 2005; 4:383 403.

2. Dawbarn D, Allen SJ. Neurotrophins and neurodegeneration. Neuropathol Appl Neurobiol 2003; 29:211 230.

3. Lang UE, Jockers-Scherubl MC, Hellweg R. State of the art of the neurotrophin hypothesis in psychiatric disorders: implications and limitations. J Neural Transm 2004; 111:387 411.

4. Gillespie LN. Regulation of axonal growth and guidance by the neurotrophin family of neurotrophic factors. Clin Exp Pharmacol Physiol 2003; 30:724 733 .

5. Lim KC, Lim ST, Federoff HJ. Neurotrophin secretory pathways and synaptic plasticity. Neurobiol Aging 2003; 24:1135 1145.

6. Eriksdotter JM, Nordberg A, Amberla K, Backman L, Ebendal T, Meyerson B, et al. Intracerebroventricular infusion of nerve growth factor in three patients with Alzheimer's disease. Dement Geriatr Cogn Disord 1998; 9:246 257.

7. Fyfe JA, Beauchamp LM, Caggiano AO, Price RD, Yamaji T, Matsuoka N, et al. KP544 amplifies the effects of nerve growth factor on cell differentiation and is neuroprotective. Drug Dev Res 2004; 62:49 59.

8. Flagstad P, Mork A, Glenthoj BY, van Beek J, Michael-Titus AT, Didriksen M. Disruption of neurogenesis on gestational day 17 in the rat causes behavioral changes relevant to positive and negative schizophrenia symptoms and alters amphetamine-induced dopamine release in nucleus accumbens. Neuropsychopharmacology 2004; 29:2052 2064.

9. Leist M, Volbracht C, Fava E, Nicotera P. 1-Methyl-4-phenylpyridinium induces autocrine excitotoxicity, protease activation, and neuronal apoptosis. Mol Pharmacol 1998; 54:789 801.

10. Falsig J, Porzgen P, Lotharius J, Leist M. Specific modulation of astrocyte inflammation by inhibition of mixed lineage kinases with CEP-1347. J Immunol 2004; 173:2762 2770.
11. Nicklas WJ, Vyas I, Heikkila RE. Inhibition of NADH-linked oxidation in brain mitochondria by 1-methyl-4-phenyl-pyridine, a metabolite of the neurotoxin, 1-methyl-4-phenyl-1,2,5,6-tetrahydropyridine. Life Sci 1985; 36:2503 2508.

12. Heneka MT, Sastre M, Dumitrescu-Ozimek L, Dewachter I, Walter J, Klockgether T, et al. Focal glial activation coincides with increased BACE1 activation and precedes amyloid plaque deposition in APP[V717I] transgenic mice. J Neuroinflammation 2005; 2.

13. Rozemuller AJ, van Gool WA, Eikelenboom P. The neuroinflammatory response in plaques and amyloid angiopathy in Alzheimer's disease: therapeutic implications. Curr Drug Targets CNS Neurol Disord 2005; 4:223 233.

14. Fiore M, Grace AA, Korf J, Stampachiacchiere B, Aloe L. Impaired brain development in the rat following prenatal exposure to methylazoxymethanol acetate at gestational day 17 and neurotrophin distribution. Neuroreport 2004; 15:1791 1795.

15. Lipton SA, Rosenberg PA. Excitatory amino acids as a final common pathway for neurologic disorders. N Engl J Med 1994; 330:613 622.

16. Beal MF. Mitochondria, free radicals, and neurodegeneration. Curr Opin Neurobiol 1996; 6:661 666.

17. Cacquevel M, Lebeurrier N, Cheenne S, Vivien D. Cytokines in neuroinflammation and Alzheimer's disease. Curr Drug Targets 2004; 5:529 534 .

18. Holmlund L, Cortes TV, Iverfeldt K. Additive effects of amyloid beta fragment and interleukin-1beta on interleukin-6 secretion in rat primary glial cultures. Int J Mol Med 2002; 10:245 250.

19. Krenitsky TA, Dillberger J, Zotova E, Arezzo JC, Koprich JB, Mortazavi F, et al. KP544, a nerve growth factor amplifier: pharmacokinetics, safety and efficacy in the rat. Drug Dev Res 2004; 62:60 70 .

20. Flagstad P, Glenthoj BY, Didriksen M. Cognitive deficits caused by late gestational disruption of neurogenesis in rats: a preclinical model of schizophrenia. Neuropsychopharmacology 2005; 30:250 260.

21. Gourevitch R, Rocher C, Le Pen G, Krebs MO, Jay TM. Working memory deficits in adult rats after prenatal disruption of neurogenesis. Behav Pharmacol 2004; 15:287 292.

22. Lavin A, Moore HM, Grace AA. Prenatal disruption of neocortical development alters prefrontal cortical neuron responses to dopamine in adult rats. Neuropsychopharmacology 2005; 30:1426 1435.

23. Penschuck S, Flagstad P, Didriksen M, Leist M, Michael-Titus AT. Decrease in parvalbumin-expressing neurons in the hippocampus and increased phencyclidine-induced locomotor activity in the rat methylazoxymethanol (MAM) model of schizophrenia. Eur J Neurosci 2006; 23:279 284 .

24. Cimino M, Cattabeni F, Di Luca M, Peruzzi G, Andena M, Tirassa P, et al. Levels of NGF, p75 NGFR and ChAT immunoreactivity in brain of adult and aged microencephalic rats. Neurobiol Aging 1996; 17:137 142. 